\title{
MISSIONE SPAZIALE PLANCK: VERSO L'ALBA DEL TEMPO
}

\author{
Nota del s.c. MARCO BERSANELLI (*)
}

(Adunanze del 22 marzo 2012 e del 19 dicembre 2013)

\begin{abstract}
SuNTO. - L'immagine più profonda dell'universo a noi accessibile proviene da osservazioni del fondo cosmico a microonde $(\mathrm{CMB})$, la radiazione elettromagnetica rilasciata quando l'età dell'universo era soltanto lo $0.003 \%$ del suo valore attuale. La missione spaziale Planck dell'Agenzia Spaziale Europea, lanciata nel 2009, ha prodotto la mappa più dettagliata mai ottenuta della CMB. I due strumenti criogenici a bordo del satellite coprono un'ampia regione spettrale ( 9 bande di frequenza fra 30 e $857 \mathrm{GHz}$ ) per assicurare un'accurata separazione della radiazione cosmologica da emissioni locali. I risultati dell'analisi dei dati di Planck sono in eccellente accordo con le predizioni del modello cosmologico standard CDM determinato da soli 6 parametri. La misura completa dello spettro angolare di potenza delle anisotropie (per tutti i multipoli $2<\ell<2500$ ) ha consentito di estrarre con grande precisione $(\sim 1 \%)$ il valore dei parametri cosmologici fondamentali che determinano la composizione, l'espansione e la geometria dell'universo. I risultati menzionati in questa discussione sono stati pubblicati dalla Collaborazione Planck nel 2014, e si riferiscono alla missione nominale (primi 15.5 mesi di osservazione in temperatura), mentre l'analisi dell'intera missione (4 anni in temperatura e polarizzazione) è attualmente in corso.
\end{abstract}

$$
* * *
$$

ABSTRACT. - The deepest image of the universe accessible to our observation comes from the cosmic microwave background $(\mathrm{CMB})$, the electromagnetic radiation released when the age of the universe was only $0.003 \%$ of its present value. The European Space Agency Planck mission, launched in 2009, has produced a full-sky map of the CMB with an unprecedented level of detail. The two cryogenic instruments on board the satellite cover a wide spectral region ( 9 frequency bands between 30 and $857 \mathrm{GHz}$ ) to ensure an accurate separation of the cosmological radiation from local foreground emissions. The results of

(") Dipartimento di Fisica, Università degli Studi di Milano, Italy.

E-mail: marco.bersanelli@unimi.it 
the Planck data analysis are in excellent agreement with the standard cosmological model CDM, which is specified by just 6 parameters. The accurate measurement of the full angular power spectrum of the anisotropies (all multipoles $2<\ell<2500$ ) has allowed a high precision $(\sim 1 \%)$ extraction of the cosmological parameters that determine the composition, the expansion, and geometry of the universe. The results mentioned in this discussion have been published by the Planck Collaboration in 2014, and refer to the nominal mission (the first 15.5. months of observation in temperature), while the analysis of the full mission (4 years in temperature and polarization) is currently in progress.

\section{INTRODUZIONE}

Nel 1922 Edwin Hubble fu in grado per la prima volta di stimare la distanza della nebulosa di Andromeda dimostrando che essa si trova ben oltre i confini della Via Lattea: fu il primo balzo nello spazio extragalattico. Pochi anni dopo emersero i primi indizi di un sistematico redshift nello spettro delle galassie, e presto si giunse alla scoperta della relazione lineare tra velocità di recessione e distanza delle galassie, indicativa dell'espansione dell'universo (Lemaitre 1927, Hubble 1929). Tutto ciò era destinato a incontrarsi splendidamente con le soluzioni cosmologiche della teoria della relatività generale, ricavate da Alexander Friedmann pochi anni prima, le quali accoglievano in maniera naturale l'espansione osservata. Nasceva così la cosmologia moderna. Nel giro di poco più di un decennio i pochi addetti ai lavori dell'epoca si resero conto che viviamo in un universo immenso e in espansione descritto con rigore ed eleganza dalla teoria della relatività generale di Einstein.

Quei primi passi della cosmologia sul terreno solido della fisica permisero di formulare alcune domande fondamentali che hanno ne accompagnato i progressi osservativi e teorici fino ad oggi: Qual è la dinamica dell'espansione cosmica? Qual è il suo futuro? Qual è l'età dell'universo? Lo spazio è euclideo oppure ha una curvatura, e di quale tipo? Quali sono i costituenti della materia e dell'energia nell'universo? Qual è il grado di isotropia su larga scala? Come si sono formate le galassie e le altre strutture che osserviamo nell'universo attuale?

\section{VERSO L'ALBA DEL TEMPO}

Oggi siamo in grado di osservare galassie a distanze ben maggiori di quelle accessibili a Hubble e ai suoi contemporanei, e abbiamo un'idea molto più precisa della distribuzione della materia su larga scala e 
dei relativi campi di velocità. Survey su ampie zone di cielo, come la Solan Digital Sky Survey (SDSS) o la $2 d F$ Redshift Galaxy Survey (2dFRGS) hanno osservato centinaia di migliaia di galassie misurando per ciascuna le coordinate sulla sfera celeste e la distanza, producendo mappe tridimensionali su regioni di spazio statisticamente significative (York et al. 2000, Percival et al. 2002). Le osservazioni mostrano che le galassie tendono a raggrupparsi in ammassi, le strutture legate più grandi nell'universo, ciascuno dei quali contiene tipicamente centinaia o migliaia di galassie. Tuttavia quando ci spingiamo su scale $>10 \mathrm{Mpc} \mathrm{la}$ distribuzione ${ }^{1}$ della materia tende progressivamente all'uniformità. La missione EUCLID, selezionata dall'ESA per un lancio nel 2020 e attualmente in fase di sviluppo, fornirà dati spettroscopici e fotometrici su campioni di decine di milioni di galassie e quasars.

Poiché la velocità della luce pur essendo grande non è infinita, quando osserviamo galassie molto distanti andiamo a sondare l'universo in tempi remoti nel passato. Poiché l'universo appare sostanzialmente isotropo e uniforme a grandi scale, osservazioni a diverse distanze in una direzione generica ci mostrano l'evoluzione delle proprietà medie dell'universo nel corso della sua storia. Il telescopio spaziale Hubble (HST) ci ha regalato le più profonde immagini dell'universo in luce visibile e infrarossa mai realizzate. L'immagine Hubble Ultra Deep Field (HUDF) realizzata con la Advanced Camera For Surveys, mostra circa diecimila galassie collocate in un'area angolare di cielo di appena 11.5 arcmin (Fig. 1). Questa immagine contiene le più lontane galassie conosciute, la cui luce ha viaggiato 12-13 miliardi di anni prima di giungere a noi, e quindi ci dà un assaggio di come era l'universo 12 o 13 miliardi di anni fa.

Poiché lo spazio è in espansione le proprietà globali dell'universo, come la densità media della materia-energia o la temperatura, variano nel tempo. L'inverso del tasso attuale di espansione, detta costante di Hubble $\left(H_{0} \approx 70 \mathrm{~km} \mathrm{~s}^{-1} \mathrm{Mpc}^{-1}\right)$, ci dà l'ordine di grandezza del tempo nel passato nel quale la distanza tra due punti qualunque dello spazio tendeva a zero, ovvero dell'età dell'universo. Più precisamente, grazie alla misura della costante di Hubble e del contributo dei diversi tipi di materia e energia, che agiscono in modo differente sulla dinamica dell'espansione, stimiamo l'età dell'universo in circa $t_{0} \approx 14$ miliardi di anni.

\footnotetext{
${ }^{1}$ Un Megaparsec $(\mathrm{Mpc})$ è $10^{6}$ parsec $(\mathrm{pc})$. Il parsec $(1 \mathrm{pc}=3.26$ anni luce= $\left.3.086 \times 10^{6} \mathrm{~m}\right)$.
} 
Poiché sia $t_{0}$ che la velocità della luce $c$ hanno valori finiti esiste un orizzonte ultimo che definisce la porzione di universo a noi accessibile, e cioè la sfera che ci circonda di raggio $t_{0} c$. Le più lontane galassie del HUDF, dunque, si trovano piuttosto vicine a quell'orizzonte, in un universo di appena 1 o 2 miliardi di anni. Ma è possibile osservare luce proveniente da regioni ancora più remote, tale che ci porti informazioni da un'epoca più prossima alle origini? Sorprendentemente, la risposta è sì. La chiave si trova sotto i nostri occhi: lo sfondo nero della volta celeste.

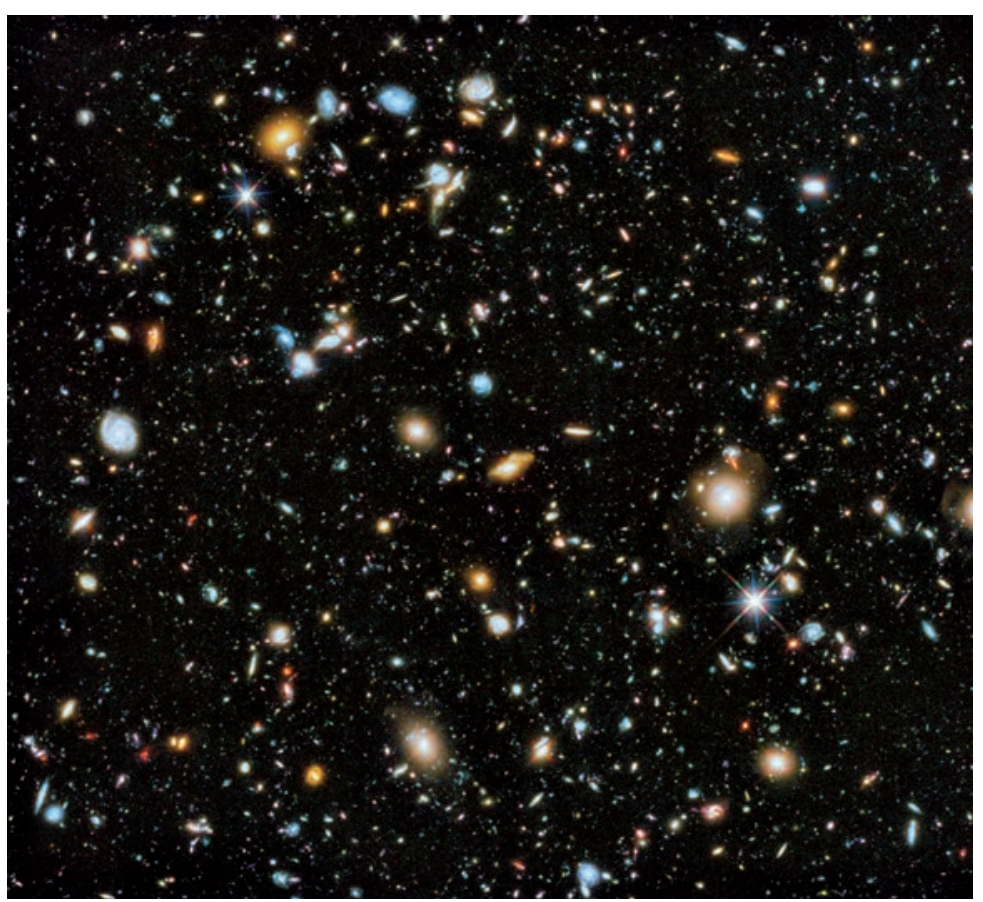

Fig. 1 - L'immagine "Hubble Ultra Deep Field" contiene più di 10 mila galassie in un'area di cielo di $2.5 \times 2.5$ arco-minuti quadrati: per coprire l'intera sfera celeste ci vorrebbero all'incirca 13 milioni di tali immagini.

Circa 50 anni fa Arno Penzias e Robert Wilson scoprirono che il fondo del cielo non è in realtà completamente oscuro, ma emana una debolissima luminosità diffusa che ci raggiunge da tutte le direzioni (Penzias \& Wilson 1965). Questa luce primordiale, che ha lunghezze 
d'onda di qualche millimetro, è chiamata fondo cosmico a microonde (Cosmic Microwave Background, CMB) ed è ciò che resta della condizione di alta temperatura e densità in cui l'universo si trovava 14 miliardi di anni fa. Quando l'età dell'universo raggiunse i 380 mila anni (solamente allo $0.003 \%$ dell'età attuale) l'espansione portò la temperatura al di sotto dei $3000 \mathrm{~K}$, e gli elettroni e i nuclei leggeri presenti nel plasma primordiale poterono per la prima volta combinarsi a formare atomi neutri (la cosiddetta "ricombinazione"). Quasi d'improvviso la materia diventò elettricamente neutra e l'universo divenne trasparente alla radiazione, la quale incominciò a propagarsi liberamente nello spazio. Era la nascita della prima luce, la CMB. A quell'epoca la lunghezza d'onda dei fotoni era di circa $0.5-1 \mu \mathrm{m}$ (visibile e vicino infrarosso) $\mathrm{ma}$ oggi, dopo 14 miliardi di anni, l'espansione ha "stirato" la lunghezza d'onda dei fotoni di un fattore 1100 spostandoli nelle microonde. Lo spettro della $\mathrm{CMB}$ che oggi osserviamo è un corpo nero pressoché perfetto ad una temperatura di $T_{0}=1.725 \pm 0.002 \mathrm{~K}$ (Mather et al. 1999). Per questo motivo il cielo notturno ci appare nero.

\section{CiRCONDATI DALLA LUCE}

A dispetto dell'enorme numero di galassie nell'universo le distanze fra loro sono enormi e lo spazio è sostanzialmente vuoto. Di conseguenza i fotoni $\mathrm{CMB}$ hanno viaggiato praticamente indisturbati e ci offrono un'immagine fedele dell'universo all'epoca della ricombinazione. La regione dalla quale noi vediamo emergere i fotoni è chiamata "superficie di ultima diffusione", una sorta di fotosfera cosmica oltre la quale la temperatura e la densità erano tali che lo scattering Thomson riduceva la materia allo stato di un plasma opaco (Fig. 2).

L'intensità della $\mathrm{CMB}$ ci appare quasi isotropa su tutto il cielo, ma non perfettamente, perché risulta increspata da lievi irregolarità. Questo leggero livello di anisotropia era stato previsto su basi teoriche perché ci si era resi conto che il processo di formazione delle galassie sotto l'azione della gravità doveva aver preso le mosse da perturbazioni di densità già presenti all'epoca dell'ultima diffusione. Poiché i fotoni sono influenzati dal potenziale gravitazionale la loro intensità necessariamente risente delle perturbazioni di densità all'epoca della ricombinazione. La presenza di anisotropie nella CMB fu osservata per la prima volta nel 1992 dallo strumento Differential Microwave Radiometer 
(DMR) a bordo del satellite della NASA COBE, che misurò fluttuazioni di ampiezza $\sim 30 \mu \mathrm{K}$ (ovvero un livello di $\sim 0.001 \%$ ) su tutte le scale angolari $>7^{\circ}$ (Smoot et al. 1992). Fu una scoperta di straordinaria importanza. Essa diede inizio ad una formidabile escalation di nuovi esperimenti da terra, da pallone stratosferico, e ben due ulteriori missioni spaziali.

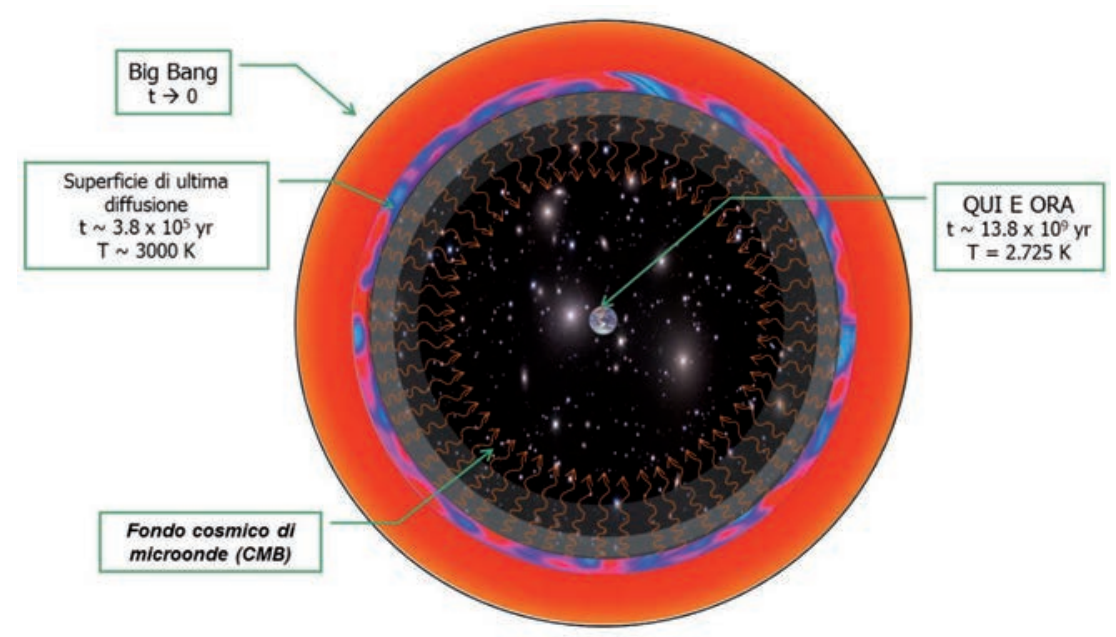

Fig. 2 - Schema spazio-temporale dell'universo osservabile come si presenta alla nostra osservazione.

Tale enorme sforzo sperimentale è giustificato dal fatto che le anisotropie della CMB sono una vera e propria miniera d'oro di informazioni cosmologiche. Se infatti dai tempi di Lemaittre e di Hubble enormi progressi sono stati fatti, le grandi domande alle quali abbiamo accennato sono rimaste aperte nei decenni sfidando generazioni di cosmologi. Ebbene, l'osservazione ad alta precisione della $\mathrm{CMB}$ è l'arma più appuntita a nostra disposizione per tentare di rispondere a quelle domande. In particolare misure ad alta risoluzione delle anisotropie della CMB forniscono informazioni dirette su proprietà fondamentali dell'universo quali la curvatura dello spazio, i costituenti di materia e energia, la costante di Hubble, e molte altre ancora. La condizione privilegiata della $\mathrm{CMB}$ in cosmologia è basata su due aspetti. Anzitutto la fisica che governa l'universo primordiale è basata su processi lineari, e quindi è molto più semplice di quella richiesta per descrivere l'universo 
evoluto di oggi. In secondo luogo la tecnologia delle microonde ha visto progressi straordinari negli ultimi decenni, consentendo misure a livello di una parte su un milione.

\section{MUSICA PRIMORDIALE}

Senza entrare in eccessivi dettagli è possibile farsi un'idea intuitiva del motivo per cui la statistica delle anisotropie CMB è rivelatrice dei parametri cosmologici fondamentali. Immaginiamo che in un certo volume nel plasma primordiale vi sia una fluttuazione di densità in eccesso (oppure in difetto) rispetto al valore medio. L'evoluzione di quella fluttuazione dipenderà dall'azione combinata di due forze: l'auto-gravità, che tenderà a comprimere quella regione, e la pressione di radiazione dei fotoni, che tenderà a espanderla. Il risultato è una serie di contrazioni ed espansioni acustiche, vere e proprie onde sonore che attraversavano il plasma: una sorta di musica cosmica dalla quale si sono formate tutte le strutture dell'universo. Tali oscillazioni acustiche continueranno fino a che, al momento della ricombinazione, la materia diventa neutra e i fotoni si disaccoppiano. Ogni fluttuazione quindi emergerà alla superficie di ultima diffusione con una certa fase di densità e di velocità.

Supponiamo ora che l'universo inizialmente (diciamo a una frazione di secondo dopo il big bang) presenti fluttuazioni su tutte le scale dimensionali. Ci domandiamo, quando avranno inizio le oscillazioni? Affinché le forze in gioco (gravità e pressione dei fotoni) agiscano su una certa fluttuazione occorre che il volume da essa occupato sia interamente contenuto all'interno dell'orizzonte causale, cioè della distanza che la luce ha potuto percorrere dal big bang fino a quell'istante. L'orizzonte causale, naturalmente, aumenta nel tempo. Di conseguenza le prime fluttuazioni che iniziano a oscillare saranno quelle di dimensioni più piccole, via via fino alle più grandi, man mano che il tempo passa. Poiché la fisica è la stessa ovunque nell'universo, le fluttuazioni di una certa dimensione entreranno nell'orizzonte causale tutte assieme, ovunque si trovino, ed emergeranno alla ricombinazione in modo sincronizzato. A seconda della loro dimensione fisica, quindi, le fluttuazioni si presenteranno all'ultima diffusione con fasi diverse e daranno luogo a una varianza di densità più o meno marcata. Le fluttuazioni che hanno avuto tempo di compiere un numero intero di espansioni o contrazioni dal big bang alla ricombinazione si presenteranno con valori massimi di 
varianza di densità all'ultima diffusione. Poiché i fotoni della CMB risentono della densità della materia alla ricombinazione, nelle mappe osservate ci aspettiamo un grado di anisotropia con dei massimi in corrispondenza di tali scale angolari. Le oscillazioni comportano anche velocità di contrazione e di espansione che, a loro volta, attraverso l'effetto Doppler incidono sull'energia dei fotoni CMB. Proprio come in un'onda sonora, le oscillazioni di velocità sono in opposizione di fase rispetto alle oscillazioni di densità.

La Fig. 3 illustra schematicamente questa situazione. Nella parte inferiore della figura fluttuazioni sono rappresentate in ordine di dimensione crescente da destra verso sinistra. Le oscillazioni entrano nell'orizzonte causale in tempi diversi a seconda delle loro dimensioni, poi iniziano ad oscillare, ed emergono alla superficie di ultima diffusione con fasi diverse. La ricombinazione non è istantanea, e ciò equivale a dire che la superficie di ultimo scattering ha uno "spessore" finito nello spazio-tempo, corrispondente a una scala angolare di $\sim 5$ arcmin. Ciò implica che le fluttuazioni di densità su scale angolari $<5$ ' presenti sulla stessa linea di vista tenderanno a compensarsi, sopprimendo rapidamente la potenza delle anisotropie (Silk damping).

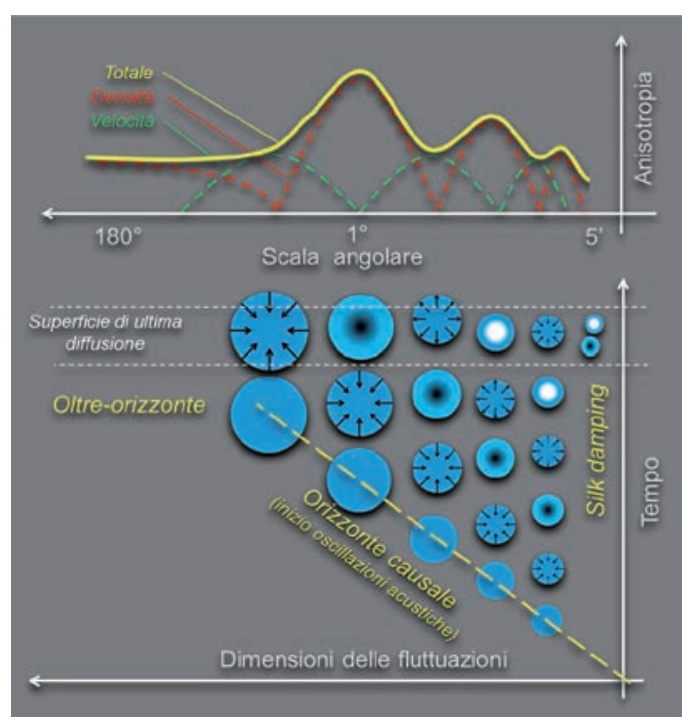

Fig. 3 - Schema rappresentativo delle oscillazioni acustiche (in basso) e dello spetrro angolare di potenza della CMB (in alto). 
La parte superiore della figura mostra è schema qualitativo dello spettro di potenza, ovvero l'andamento della potenza delle anisotropie (il contrasto o varianza tra regioni di intensità diversa) in funzione della scala angolare. I contributi parziali di densità (curva rossa) e di velocità (curva verde) sono sfasati, e la loro combinazione genera lo spettro complessivo misurato (curva gialla). Lo spettro di potenza rappresenta la sintesi della statistica delle oscillazioni acustiche su tutte le scale angolari $\theta$, e viene normalmente rappresentato in funzione dei multipoli $\ell \approx \pi / \theta$. In termini quantitativi possiamo descrivere le anisotropie come variazioni di temperatura equivalente $\Delta T$ sulla superficie di ultima diffusione in funzione delle armoniche sferiche $Y_{\ell m}$ :

$$
\Delta T(\theta, \varphi)=\sum_{\ell, m} a_{\ell m} Y_{\ell m}(\theta, \varphi)
$$

Per una distribuzione Gaussiana le proprietà statistiche sono riducibili in modo esatto allo spettro di potenza definito da

$$
C_{\ell}=\left\langle\left|a_{\ell m}\right|^{2}\right\rangle=\frac{1}{2 \ell+1} \sum_{m=-\ell}^{\ell} a_{\ell m}^{2}
$$

In Fig. 3, come è consuetudine, le anisotropie sono rappresentate dalla funzione $\ell(\ell+1) C_{\ell}$, che produce una curva piatta a grandi scale angolari.

Il picco principale dello spettro di potenza (il primo da sinistra) corrisponderà a fluttuazioni che hanno avuto appena il tempo di oscillare (di comprimersi o di rarefarsi) una sola volta dall'inizio dell'oscillazione fino alla ricombinazione. Quelle fluttuazioni avranno quindi le dimensioni dell'orizzonte causale alla superficie di ultima diffusione. Poiché ciò avvenne 380 mila anni dopo il big bang, le loro dimensioni tipiche saranno 380 mila anni luce. E poiché noi osserviamo questa scena da una distanza di $~ 14$ miliardi di anni luce e dopo una espansione di un fattore $z \sim 1100$, siamo in grado di calcolare qual è la dimensione angolare nel cielo a cui ci aspettiamo di osservare il primo picco: questa corrisponde a circa $1^{\circ}$, ovvero $\ell \approx 200$.

Ma come tutto ciò può darci informazioni sui parametri cosmologici? Vediamo qualche esempio. Nella precedente discussione abbiamo calcolato la scala angolare come rapporto fra la dimensione delle oscillazioni massime e la distanza della superficie di ultima diffusione. $\mathrm{Ma}$ questo è vero solo se lo spazio è Euclideo (il che accade quando la densità di massa-energia $\rho$ è pari al valore critico $\left.\rho_{0}=3 H_{0}^{2} / 8 \pi G\right)$. Se invece 
lo spazio ha una curvatura positiva $\left(\rho>\rho_{0}\right)$ le dimensioni delle oscillazioni massime sottenderanno un angolo $>1^{\circ}$, proprio come una distanza lineare su una superficie sferica sottende un angolo maggiore a quello che avremmo su a una superficie piana. Viceversa l'angolo sarà $<1^{\circ}$ per una curvatura negativa $\left(\rho<\rho_{0}\right)$. Una misura della scala angolare del primo picco, quindi, permette di stabilire la curvatura dell'universo e la densità media di massa-energia $\rho$.

Inoltre la propagazione delle oscillazioni acustiche dipende in modo importante e predicibile dalle proprietà fisiche del plasma primordiale. In particolare a seconda della miscela dei diversi tipi di materia e energia presenti nel plasma ci aspettiamo di osservare uno spettro di potenza con picchi di ampiezze e posizioni diverse. Ad esempio, poiché la materia oscura contribuisce alla gravità ma non alla pressione dei fotoni, essa alimenta la dinamica delle oscillazioni acustiche in modo differente rispetto alla materia barionica, la quale contribuisce a entrambe le interazioni. Analogamente la densità di energia oscura o il numero e la massa dei neutrini modificano lo spettro di potenza in modo più lieve ma ben compreso dalla teoria. Il valore di $H_{0}$ invece determina la scala delle dimensioni e quindi una dislocazione generale delle scale angolari osservate.

In definitiva la posizione e ampiezza dei picchi su scale angolari $5^{\prime}<\theta<1^{\circ}$ contengono informazioni dirette sulla composizione, la geometria e l'espansione dell'universo. La Fig. 4 esemplifica la sensibilità dello spettro di potenza a due parametri cosmologici, la densità barionica $\Omega_{b}$ e la costante di Hubble $h=H_{0} / 50 \mathrm{~km} \mathrm{~s}^{-1} \mathrm{Mpc}^{-1}$.
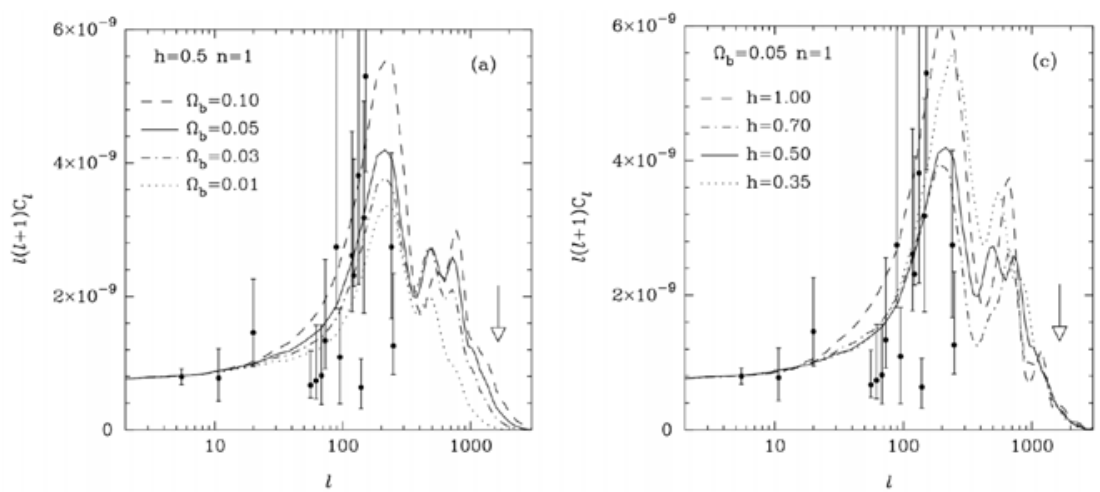

Fig. 4 - Esemplificazione della sensibilità dei dettagli dello spettro di potenza al variare dei parametri cosmologici. A sinistra: componente barionica $\Omega_{\mathrm{b}}$ espressa in termini di densità critica. A destra: costante di Hubble $b \equiv H_{0} / 50 \mathrm{~km} \mathrm{~s}^{-1} \mathrm{Mpc}^{-1}$. 
Che cosa succede alle fluttuazioni su scale angolari $\theta>1^{\circ}$ ? Esse non hanno avuto tempo di entrare nell'orizzonte cosmico prima della ricombinazione e quindi non hanno subito oscillazioni. Esse hanno quindi attraversato indisturbate tutta la fase di plasma dell'universo, e ci presentano uno scorcio pressoché intatto delle fluttuazioni originarie. Sono in assoluto gli "oggetti" cosmici più antichi accessibili alla nostra indagine. Ma qui si evidenzia un problema: se le regioni a scale angolari $>1^{\circ}$ all'ultima diffusione erano prive di contatto causale, come mai mostrano una sostanziale isotropia (a un livello 0.001\%)? Torneremo su questa domanda cruciale.

La Fig. 4 è tratta dallo studio di Fase A del progetto Planck, allora denominato COBRAS/SAMBA, quando la missione fu selezionata dall'Agenzia Spaziale Europea (Bersanelli et al. 1996). I punti sperimentali mostrano i migliori dati allora disponibili, ancora insufficienti per evidenziare i picchi acustici e ben lontani dal discriminare i diversi valori dei parametri. Successivamente, a partire dal 2000, lo spettro di potenza su scale angolari $<1^{\circ}$ fu misurato su regioni limitate del cielo da diversi esperimenti da terra e da pallone stratosferico (a cominciare da Boomerang, De Bernardis et al. 2000, Lange et al. 2001) che hanno confermato la presenza dei picchi acustici ed iniziato ad usarli per vincolare i parametri cosmologici. In particolare la misura della posizione del primo picco indicava che lo spazio è con buona approssimazione Euclideo. Le misure del satellite WMAP, lanciato dalla NASA nel 2000, dopo nove anni di osservazioni hanno prodotto mappe della CMB su tutto il cielo in 5 bande di frequenza fra 23 e $90 \mathrm{GHz}$, confermando e migliorando i vincoli cosmologici (Hinshaw et al. 2013). Recentemente i telescopi SPT (Reichardt et al. 2012) e ACT (Das et al. 2014) hanno misurato le anisotropie CMB ad alta risoluzione angolare, misurando i picchi successivi dello spettro di potenza. La teoria delle oscillazioni del plasma primordiale, introdotta fin dagli anni '80, è stata brillantemente confermata dalle osservazioni. Nel 2009, infine, l'ESA ha lanciato il satellite Planck, il cui sviluppo ha richiesto 17 anni di lavoro, e i cui risultati appena resi pubblici hanno portato la cosmologia ad un nuovo livello di precisione.

\section{IL FONDO POLARIZZATO}

Un'altra fonte straordinaria di informazione che ci offre la CMB viene dal suo grado di polarizzazione. Lo scattering Thomson alla 
superficie di ultima diffusione, in presenza di una componente di quadrupolo dell'anisotropia, induce un certo grado di polarizzazione lineare nei fotoni CMB. La prima rivelazione di tale polarizzazione, al livello del $10 \%$ come previsto dalla teoria, è stata ottenuta dall'esperimento DASI (Kovac et al. 2002) e in seguito da WMAP e da numerosi altri esperimenti. Tali anisotropie in polarizzazione (dette "modi E") essendo causate dalle stesse fluttuazione di densità che generano le anisotropie in temperatura, presenteranno uno spettro di potenza correlato a quello discusso in Fig. 3, e che a sua volta dipenderà dal valore dei parametri cosmologici. Una misura precisa dello spettro dei modi E della polarizzazione, quindi, fornisce un metodo per migliorare ulteriormente la conoscenza dei parametri fondamentali.

L'osservazione dei picchi nello spettro di potenza in temperatura conferma che inizialmente l'universo conteneva fluttuazioni su tutte le scale. Ma subito si aprono altre domande: qual è l'origine di tali fluttuazioni primordiali? E come mai la CMB ci appare globalmente isotropa (a parte le fluttuazioni al livello di $0.001 \%$ ) su scale ben superiori all'orizzonte causale? E perché la geometria dello spazio risulta essere tanto vicina a quella euclidea? Quest'ultimo problema ("flatness problem") è esacerbato dal fatto che anche una infima deviazione dalla geometria euclidea nelle prime frazioni di secondo dopo il big bang condurrebbe a livelli di curvatura macroscopici nell'universo attuale.

Lo scenario attualmente più accreditato per rispondere a queste domande è l'ipotesi dell'inflazione, introdotta per la prima volta agli inizi degli anni '80. L'idea centrale è che nei primi $10^{-35}$ secondi l'espansione dell'universo sarebbe avvenuta con una crescita esponenziale (Guth 1997). Questa semplice modifica al modello classico risolverebbe il flatness problem in quanto una generica curvatura iniziale sarebbe stata diluita dall'enorme fattore di espansione iniziale. Anche il problema dell'isotropia su larga scala verrebbe superato in quanto regioni attualmente fuori contatto causale erano causalmente connesse prima dell'inflazione, e furono portate fuori orizzonte proprio dall'espansione iniziale avvenuta a un tasso superluminale. Lo scenario inoltre prevede che il seme iniziale venisse da fluttuazioni quantistiche che l'inflazione avrebbe amplificato su scala macroscopica producendo le disomogeneità responsabili delle anisotropie della $\mathrm{CMB}$ che oggi osserviamo. L'inflazione offre quindi una risposta ad alcuni problemi aperti del modello standard e suggerisce al tempo stesso un meccanismo fisico per l'origine delle fluttuazioni. 
Una delle frontiere della cosmologia attuale è tentare di fornire evidenze osservative per sostenere o confutare l'inflazione. Una difficoltà in questo senso è che esistono un gran numero di varianti della teoria dell'inflazione ed è arduo sottoporre lo scenario generale ad una effettiva eventuale falsificazione. Ma è di grande interesse sondare il terreno e delimitare le varianti compatibili con i dati. Come abbiamo visto, fra i presupposti dei modelli di inflazione vi è una curvatura piccola $\left(\Omega_{K} \approx 0\right)$. Inoltre i modelli più semplici prevedono uno spettro delle fluttuazioni primordiali all'incirca indipendente dalla scala angolare (descritta dal parametro $n_{S} \approx 1$ ) o leggermente inferiore. Sia $\Omega_{K}$ che $n_{S}$ sono misurabili dallo spettro di potenza delle anisotropie in temperatura.

Le più ambiziose possibilità di verifica dell'inflazione vengono dalla polarizzazione della CMB. I modelli di inflazione prevedono infatti che, oltre alle perturbazioni di densità, vi siano perturbazioni prodotte da onde gravitazionali generate durante l'accelerazione espansiva iniziale (Boyle et al. 2006). Distorcendo lievemente la superficie dalla quale i fotoni vengono rilasciati le onde gravitazionali generano una componente polarizzata aggiuntiva nella CMB, i cosiddetti "modi B", distinguibili statisticamente dai "modi E". L'ampiezza delle onde gravitazionali è proporzionale al quadrato della scala di energia alla quale esse sono state generate. Se fossero osservati, i modi $B$ rappresenterebbero quindi un'impronta inconfondibile dell'inflazione e ci permetterebbero di determinare la scala di energia alla quale le fluttuazioni iniziali sono state generate nelle primissime frazioni di secondo dopo il big bang.

\section{PLANCK: LA NUOVA FRONTIERA}

Il progetto Planck prese le mosse nel lontano 1992, all'indomani della prima rilevazione delle anisotropie da parte di COBE-DMR, ed è stato sviluppato con l'ambizione di estrarre in modo definitivo le informazioni cosmologiche codificate nelle anisotropie di temperatura della $\mathrm{CMB}$ e di spingere le misure di polarizzazione ben oltre i risultati precedentemente raggiunti (Planck Collaboration 2005, 2011). Il satellite Planck è stato lanciato da un Ariane 5 dalla base ESA di Kouru, nella Guiana Francese, il 14 Maggio 2009 alle 10:12 (ora locale). Da allora per 4 anni ha fornito senza interruzione dati di eccellente qualità. I risultati di cui parleremo qui sono stati pubblicati dalla nel 2014 (Planck Collaboration 2014), e si riferiscono alla missione nominale 
(primi 15.5 mesi di osservazione in temperatura), mentre l'analisi dell'intera missione (4 anni di dati sia in temperatura che in polarizzazione) è attualmente in corso.

$\mathrm{Ma}$ in che senso l'obiettivo di Planck è di ottenere una misura "definitiva" delle anisotropie in temperatura? Fino a che punto possiamo spingere la precisione delle misure dello spettro di potenza ed estrarre il valore dei parametri fondamentali? E quali limiti possiamo imporre agli scenari di inflazione?

A differenza della maggior parte dei telescopi, i quali osservano un gran numero di sorgenti celesti, missioni spaziali come COBE, WMAP e Planck osservano un singolo peculiare oggetto: l'intero cielo. Quando osserviamo il cielo nelle microonde, oltre alla CMB riceviamo radiazione anche dalla Via Lattea e da sorgenti extragalattiche, dette in gergo emissioni di "foreground". Grazie ad una fortunata coincidenza della Natura, il massimo dello spettro di corpo nero della CMB cade nei pressi del minimo della combinazione delle emissioni di foreground, intorno ai 70-100 GHz. L'estrema sensibilità di Planck, tuttavia, richiede che la contaminazione dei foreground sia rimossa con estrema cura. Per questa ragione Planck è stato progettato per osservare in ben nove bande di frequenza, fra 30 e $850 \mathrm{GHz}$, coprendo la finestra spettrale ottimale ma anche spingendosi in regimi dove le emissioni di foreground sono dominanti per poterle meglio sottrarre. Planck ha così prodotto una mappa full-sky della CMB libera da contaminazioni e, al tempo stesso, le nove mappe in frequenza le quali rappresentano una straordinaria fonte di informazioni per lo studio astrofisico della nostra Galassia e di sorgenti extragalattiche.

Per ottenere prestazioni eccellenti su un range spettrale tanto ampio è stato necessario usare due diverse tecnologie (Bersanelli et al. 2010; Lamarre et al. 2010): amplificatori coerenti a bassissimo rumore per le bande centrate a 30, 44, $70 \mathrm{GHz}$ (Low Frequency Instrument, LFI), e rivelatori bolometrici per le bande centrate a 100, 143, 217, 343, 545, $857 \mathrm{GHz}$ (High Frequency Instrument, HFI). L'architettura termica di Planck è forse l'aspetto più innovativo e rischioso del payload (Planck Collaboration 2011), con un raffreddamento passivo che porta il telescopio e il payload (in L2) a 36-42 K, più tre diversi refrigeratori in serie: un sorption cooler fornisce lo stadio a $18-20 \mathrm{~K}$ in comune ai due strumenti; uno Stirling cooler per lo stadio a $4.5 \mathrm{~K}$ a cui si trovano gli elementi passivi di HFI e i calibratori di riferimento di LFI; e un raffreddatore a diluizione ${ }^{3} \mathrm{He} /{ }^{4} \mathrm{He}$ che porta i bolometri di HFI fino a $0.1 \mathrm{~K}$ (la temperatura 
più bassa mai ottenuta nello spazio). La copertura a tutto cielo delle osservazioni di Planck consente la misura dello spettro di potenza fino ai multipoli più bassi, ovvero fino alle più grandi scale angolari. Dalla parte opposta dello spettro, per assicurare la misura di tutti i multipoli significativi dal punto di vista cosmologico, occorre spingersi fino alla scala angolare del Silk damping, che come abbiamo visto è $\sim 5$ arcmin. E' questo il requisito che ha fissato il diametro dello specchio primario del telescopio di Planck a $1.5 \mathrm{~m}$, che garantisce una risoluzione di 5' ai canali ad alta frequenza. Per quanto riguarda la sensibilità, il rumore strumentale alle temperature di operazione è talmente basso che l'errore di misura per pixel a fine missione è inferiore all'ineliminabile incertezza dovuta alla natura statistica dello spettro di potenza ("varianza cosmica"). In questo senso la misura di Planck delle anisotropie in temperatura, se non è contaminata da effetti sistematici, è "definitiva" (Fig. 5).
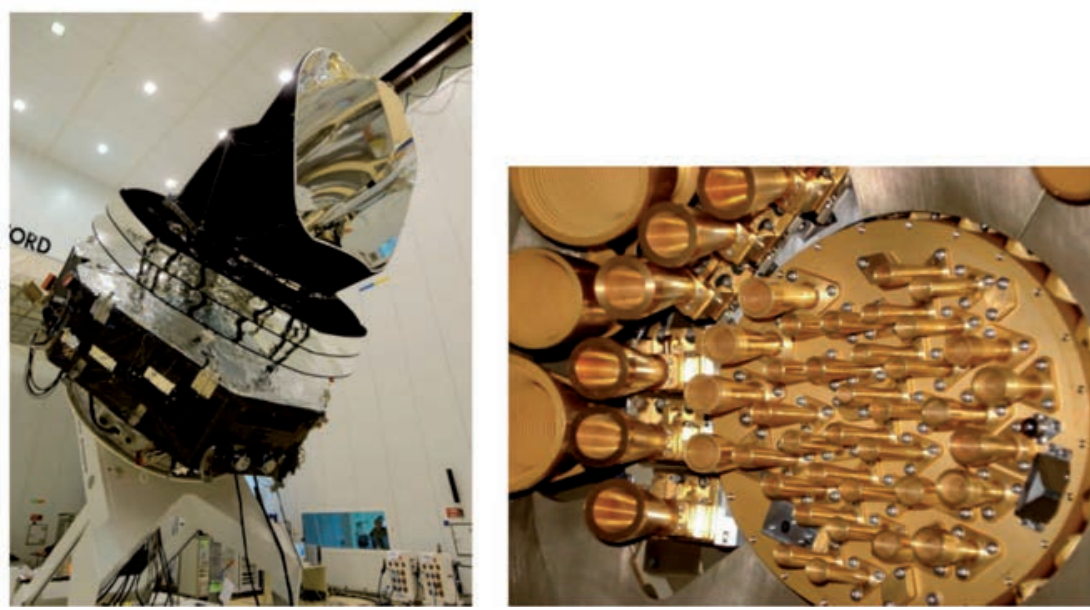

Fig. 5 - A sinistra: immagine del satellite Planck durante gli ultimi test di sistema prima del lancio. E' visibile, all'interno del grande schermo che blocca la radiazione fuori asse, lo specchio primario del telescopio (1.5 $\mathrm{m}$ di diametro).

I tre schermi orizzontali separano termicamente la parte calda (modulo di servizio, in basso) da quella criogenica. A destra: immagine del piano focale con le antenne corrugate per la rivelazione delle microonde provenienti dall'universo primordiale (ESA, ASI, CNES).

Affinché la precisione delle misure sia effettivamente limitata dalla sensibilità dei rivelatori le potenziali sorgenti di errori sistematici 
devono essere minimizzare fin dalla fase di progetto. Nel caso di Planck, l'altissima sensibilità (pochi $\mu \mathrm{K}$ per pixel) richiede che tali effetti siano soppressi a livelli estremi. Uno degli effetti più difficili da controllare è quello della radiazione che viene diffratta sul piano focale pur provenendo da direzioni diverse da quella di osservazione, la cosiddetta straylight. Per minimizzare l'effetto di straylight della Terra Planck è stato lanciato in un'orbita attorno al punto Lagrangiano L2 del sistema Terra-Sole, a 1.5 milioni di km dalla Terra, riducendo l'angolo solido della Terra di un fattore $\sim 10^{5}$. L'orbita attorno a L2 è anche favorevole dal punto di vista della stabilità termica, sfruttata appieno dal fatto che durante la survey l'asse di spin del satellite è stato mantenuto vicino alla direzione antisolare. Con una rotazione dello strumento intorno al suo asse si può esplorare il cielo lungo grandi cerchi (la direzione di osservazione del telescopio è inclinata di $85^{\circ}$ rispetto all'asse di rotazione), senza incontrare mai l'emissione della terra, della luna, e del sole. Gli effetti ottici di straylight ora citati sono solo un esempio fra i molti effetti sistematici accuratamente tenuti sotto controllo, quali rumore $1 / \mathrm{f}$, effetti dovuti a instabilità termiche, fluttuazioni elettriche, errori di puntamento, disuniformità in banda, non linearità della risposta strumentale, incertezze nella forma dei fasci d'antenna.

La campagna di test e calibrazione a terra degli strumenti e della catena criogenica è stata condotta nell'arco di oltre 10 anni, con test sulle singole unità, sugli strumenti integrati, e sull'intero satellite. Dopo il lancio, durante il lungo viaggio (due mesi) verso l'orbita in L2 il payload ha completato la procedura di raffreddamento, per poi passare alla fase di calibrazione in-flight, e infine alla survey del cielo. Ciascuna copertura dell'intera volta celeste ha richiesto circa 6 mesi, per cui l'intero cielo è stato osservato più volte, a distanza di tempo e con passaggi sui pixel in direzioni diverse, rendendo così possibile la rimozione di drift residui e l'attivazione di diversi test di auto-consistenza. Entrambi gli strumenti hanno funzionato magnificamente durante tutta la missione, con il 100\% dei canali funzionanti a temperature criogeniche $(22$ radiometri e 50 bolometri). Le prestazioni previste in fase di design e calibrazione a terra sono state pienamente confermate dalle misure in-flight. Inoltre, grazie alla straordinaria performance della catena criogenica la durata della survey è stata di molto superiore ai 15.5 mesi previsti nella missione nominale: 29 mesi per HFI e 48 mesi per LFI. La Tab. 1 mostra i dettagli delle performance osservative di Planck. Mai la prima luce dell'universo era stata osservata con tale completezza e dettaglio. 
Tab. 1 - Parametri strumentali di Planck misurati in orbita. Per ogni canale di frequenza vengono dati il numero di rivelatori (con o senza sensibilità alla polarizzazione); la risoluzione (in termini di full-width half-maximum, FWHM) e l'ellitticità dei fasci d'antenna; la larghezza di banda; la sensibilità per pixel dopo la missione nominale (15.5 mesi di osservazione), in temperature (Stokes I), in polarizzazione (Stokes $Q$ e U) e per sorgenti puntiformi.

\begin{tabular}{l|ccc|cccccc}
\hline \hline Instrument & \multicolumn{3}{|c|}{ LFI } & \multicolumn{6}{c}{ HFI } \\
\hline Center frequency [GHz] & 30 & 44 & 70 & 100 & 143 & 217 & 353 & 545 & 857 \\
Number of polarised detectors & 4 & 6 & 12 & 8 & 8 & 8 & 8 & & \\
Number of unpolarised detectors & & & & & 4 & 4 & 4 & 4 & 4 \\
Mean FWHM (arcmin) & 32.7 & 29.5 & 13.0 & 9.6 & 7.0 & 4.6 & 4.5 & 4.7 & 4.3 \\
Mean ellipticity & 1.36 & 1.50 & 1.27 & 1.17 & 1.05 & 1.11 & 1.13 & 1.03 & 1.04 \\
Bandwidth ( $\Delta v, \mathrm{GHz})$ & 4.5 & 4.1 & 12 & 32 & 45 & 68 & 104 & 174 & 258 \\
$\Delta T / T$ per pixel (Stokes $I)$ & 3.3 & 5.2 & 8.9 & 3 & 2.2 & 4.8 & 2.0 & 150 & 6000 \\
$\Delta T / T$ per pixel (Stokes $Q \& U)$ & 4.6 & 7.4 & 12.7 & 4.8 & 4.1 & 9 & 38 & & \\
Point source sensitivity $(1 \sigma, \mathrm{mJy})$ & 22 & 59 & 46 & 14 & 10 & 14 & 38 & 44 & 45 \\
\hline
\end{tabular}

Di fondamentale importanza è la calibrazione degli strumenti. Nel caso di Planck la calibrazione è basata sulla misura dell'anisotropia di dipolo indotta per effetto Doppler sul fondo di microonde a causa del moto del satellite rispetto al sistema della CMB. Tale velocità è dovuta alla composizione del moto del satellite intorno al baricentro del sistema solare e del moto del Sole rispetto al flusso di Hubble. La velocità orbitale del satellite (dominata dal moto di rivoluzione terrestre, $\sim 30 \mathrm{~km} / \mathrm{s}$ ) è conosciuta con estrema precisione, ed è questa che viene sfruttata per operare una calibrazione assoluta. Confrontando il segnale di dipolo orbitale misurato in tutti i canali da 30 a $353 \mathrm{GHz}$ con il segnale aspettato si è ottenuta la calibrazione fotometrica con precisione intorno a $\sim 0.4 \%$ (con l'analisi della missione completa si prevede di arrivare a una precisione dello $0.1 \%$ ).

\section{NUMERI COSMICI}

La Fig. 6 mostra le nove mappe del cielo ottenute da Planck in coordinate galattiche. Alle frequenza più basse $(30 \mathrm{GHz})$ e più alte $(217-857 \mathrm{GHz})$ il contributo dell'emissione galattica è molto forte. Alle frequenze centrali (44-143 GHz), specie ad alte latitudini galattiche, il segnale è invece dominato dalle anisotropie della CMB.

Come già anticipato, l'ampia copertura spettrale ha lo scopo di assicurare un'accurata rimozione delle emissioni di foreground per ottenere una mappa CMB incontaminata su tutto il cielo. Esistono 
diversi algoritmi di "separazione delle componenti", che differiscono anche radicalmente fra loro per le ipotesi di base e per le procedure matematiche utilizzate. In generale si sfrutta l'indipendenza statistica dei diversi tipi di emissione e la nostra (parziale ) conoscenza a priori della dipendenza delle varie componenti dalla lunghezza d'onda (per la $\mathrm{CMB}$ essa è nota con estrema precisione, ed è quella di un corpo nero a $2.725 \mathrm{~K})$. Nel caso di Planck si sono utilizzati 4 metodi diversi di separazione delle componenti. L'eccellente accordo tra le quattro mappe di $\mathrm{CMB}$ così prodotte dimostra l'affidabilità del risultato.

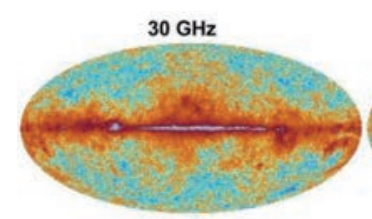

$100 \mathrm{GHz}$

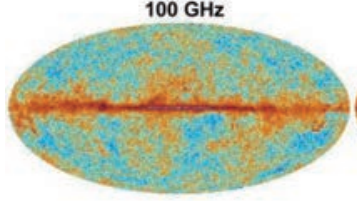

$353 \mathrm{GHz}$
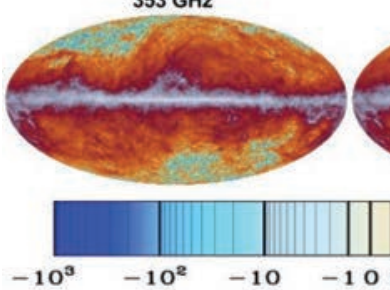

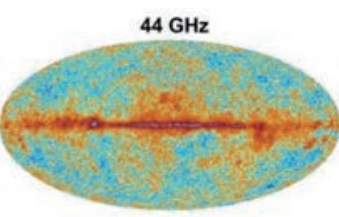

$143 \mathrm{GHz}$

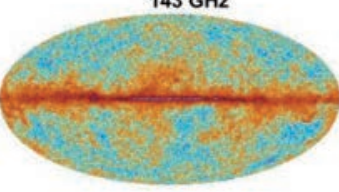

$545 \mathrm{GHz}$

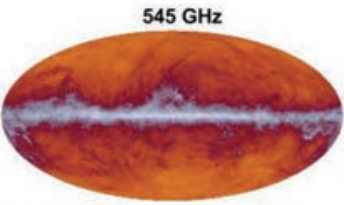

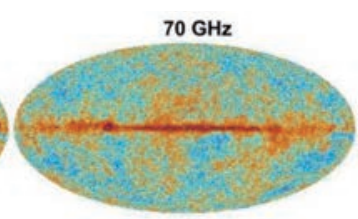

$217 \mathrm{GHz}$

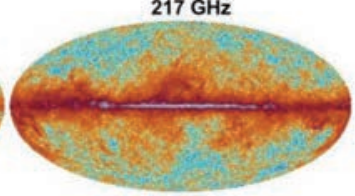

$857 \mathrm{GHz}$

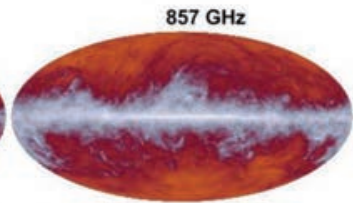

Fig. 6 - Le nove mappe in frequenza di Planck. Ciascuna mappa è costituita da circa 6 milioni di pixel indipendenti, ciascuno di 5 minuti d'arco di lato, quindi la figura non può rendere conto della ricchezza di dettagli presenti.

Il processo di separazione ha inoltre prodotto mappe full-sky delle varie emissioni, le quali rivestono grande interesse per l'astrofisica galattica ed extragalattica. E' stata così realizzata una mappa dell'emissione termica della polvere interstellare della nostra galassia, una componente decisiva per studiare il fenomeno ancora non pienamente compreso della formazione stellare. E' stata poi realizzata una mappa dell'emissione degli elettroni liberi, con le emissioni di free-free e di sincrotrone, quest'ultima fondamentale per lo studio del campo magnetico 
galattico. A basse frequenze è stata anche evidenziata una componente dovuta all'emissione di dipolo rotante dovuta al grado di libertà rotazionale di piccoli grani di polvere elettricamente carichi, la cosiddetta emissione di microonde "anomala"(AME), che presenta un picco di intensità intorno a 10-30 GHz. E' stato inoltre realizzato un catalogo di circa 15000 sorgenti puntiformi, per lo più extragalattiche, alcune delle quali con massimo di emissione nel radio, altre nell'infrarosso.

Un contributo di foreground sui generis è quello prodotto dal gas ionizzato (con temperature $\mathrm{T}_{\mathrm{e}} \sim 10^{7}-10^{8} \mathrm{~K}$ ) presente negli ammassi di galassie che perturbano i fotoni CMB che li attraversano (effetto Sunyaev-Zel'dovich, SZ). Per effetto Compton inverso i fotoni aumentano la loro energia di un fattore $\mathrm{kT}_{\mathrm{e}} / \mathrm{m}_{\mathrm{e}} \mathrm{c}^{2} \sim 1 \%$, con una probabilità di diffusione dell' $1 \%$ circa. $\mathrm{Ci}$ aspettiamo quindi di osservare in direzione di un ammasso di galassie una variazione di brillanza della CMB dell'ordine di 1 parte su 10000, 10 volte maggiore delle anisotropie intrinseche. L'effetto SZ produce una distorsione spettrale caratteristica, relativamente facile da identificare nelle mappe $\mathrm{CMB}$, con un deficit di fotoni a frequenze $<217 \mathrm{GHz}$ ed un eccesso a frequenze $>217 \mathrm{GHz}$. Trattandosi di un fenomeno di scattering, la sua entità non dipende dalla posizione dell'ammasso sulla linea di vista: ammassi lontani provocano la stessa variazione di brillanza di ammassi vicini. L'effetto SZ offre quindi la possibilità di misurare un grande numero di ammassi di galassie, e di usarli come sonde cosmologiche. La separazione delle componenti sui dati di Planck ha permesso di estrarre un catalogo di oltre 1200 candidati ammassi ricchi di galassie (vedi Fig. 7).

Ma lo scopo fondamentale, naturalmente, è l'estrazione della mappa dell'anisotropia del fondo cosmico di microonde (Fig. 8). Il numero di componenti di foreground appena elencate mostra come fosse impossibile ottenere risultati accurati con gli strumenti predecessori di Planck, che osservavano un numero di frequenze inferiore al numero di componenti indipendenti dell'emissione.

Per costruire lo spettro di potenza sono state applicate due procedure diverse a seconda della scala angolare. A gradi scale angolari (per multipoli $\ell<50$ ) si sono usate le mappe di LFI e HFI da 30 a 353 $\mathrm{GHz}$ per separare le componenti sul $87 \%$ del cielo, evitando il piano galattico. Per stimare i multipoli superiori si sono usate maschere più conservative $(58 \%$ del cielo a $100 \mathrm{GHz}, 37 \%$ del cielo a 143 e 217 $\mathrm{GHz}$ ) e si è operata un'analisi di cross-spettro, ottenuta cioè combinando i dati a frequenze diverse per minimizzare correlazioni spurie. 


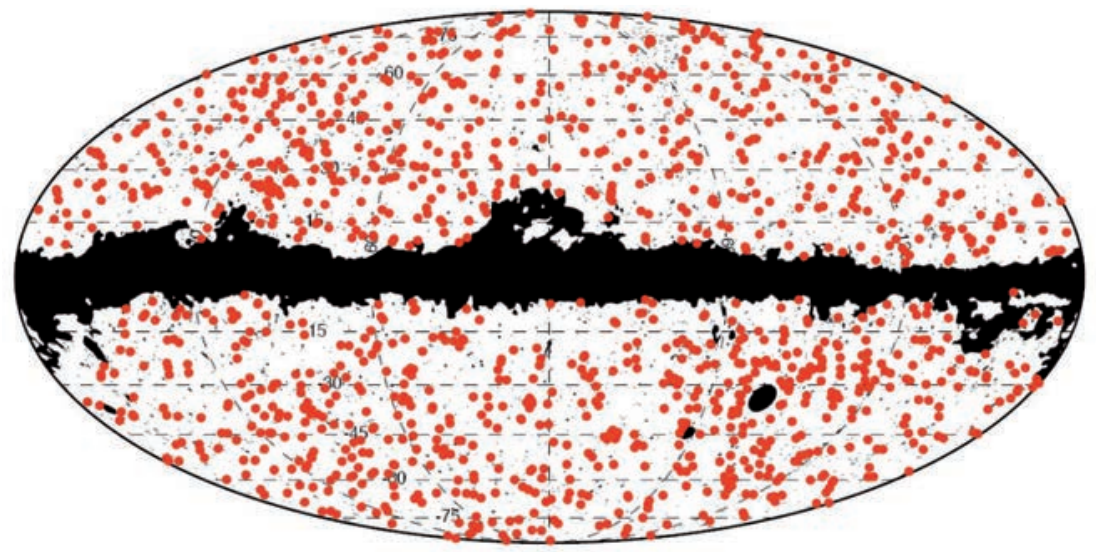

Fig. 7 - La mappa indica la posizione dei 1227 candidati ammassi di galassie osservati da Planck grazie all'effetto Sunyaev-Zel'dovich.

La regione scura indica la regione del piano galattico nella quale a causa della forte emissione diffusa l'effetto SZ non è osservabile.

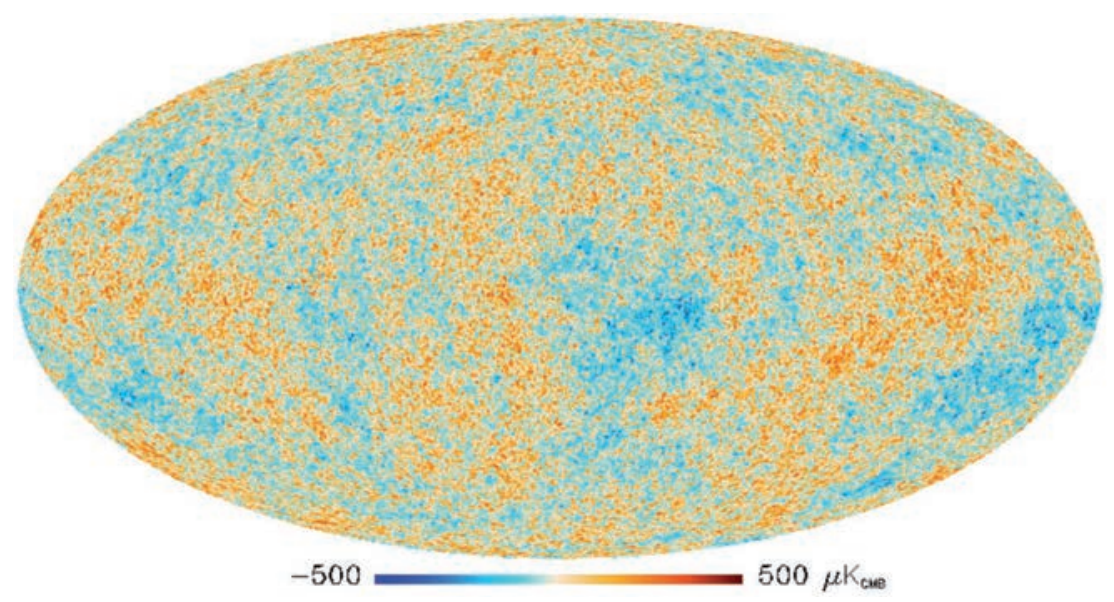

Fig. 8 - La mappa a tutto cielo del fondo cosmico di microonde estratta dalle mappe di Planck alle diverse frequenze tramite un sofisticato sistema di separazione delle componenti, che sfrutta la diversa dipendenza dalla frequenza delle diverse emissioni. L'anisotropia di dipolo dovuta al moto dell'osservatore rispetto alla materia lontana è stata sottratta. Le macchie colorate individuano regioni leggermente più fredde (blu)

o leggermente più calde (rosse) a causa delle fluttuazioni di densità presenti 13.8 miliardi di anni fa, e progenitrici delle strutture presenti oggi nell'universo. 
La Fig. 9 mostra lo spettro di potenza misurato da Planck insieme alla curva di best fit del modello standard (curva verde). L'accordo è impressionante. Il modello di riferimento, denominato CDM, assume curvatura nulla $\left(\Omega_{0}=0\right)$ con il contributo di materia barionica, di materia oscura non-relativistica (cold dark metter, CDM), e della costante cosmologica $(\Lambda)$. Il modello è completamente determinato da sei parametri indipendenti, che definiscono la composizione dell'universo e le proprietà statistiche delle fluttuazioni iniziali di densità. Una possibile scelta dei 6 parametri è la seguente: l'ampiezza globale dello spettro di potenza delle perturbazioni iniziali di densità $\left(A_{s}\right)$ e il suo indice spettrale $\left(n_{s}\right)$; lo spessore ottico $(\tau)$ attraversato dai fotoni dopo la ricombinazione, che dipende sensibilmente dall'epoca in cui l'universo si reionizza a causa della formazione delle prime stelle; le dimensioni angolari sottese dall'orizzonte alla ricombinazione $\left(\theta_{\mathrm{MC}}\right)$; e i contributi alla densità totale da parte dei barioni $\left(\Omega_{b} h^{2}\right)$ e della materia oscura $\left(\Omega_{c} b^{2}\right)$. Gli altri parametri cosmologici (la costante di Hubble, la densità di energia oscura, l'età dell'universo, etc.) possono essere derivati da combinazioni di questi sei parametri.

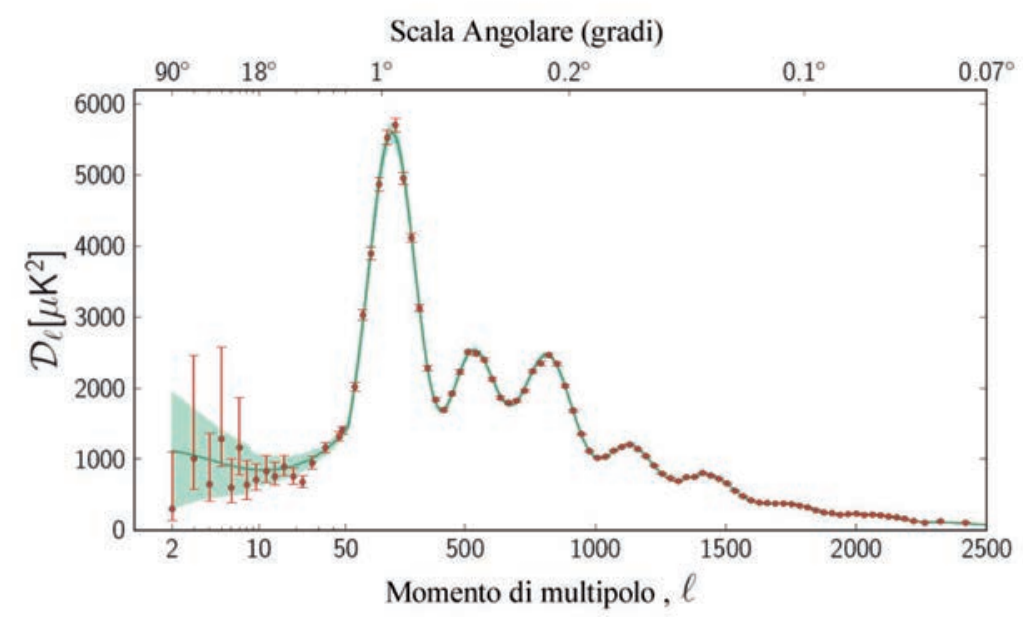

Fig. 9 - Spettro di potenza dell'anisotropia della CMB misurato dal satellite Planck. Le barre d'errore includono sia l'errore dovuto alla varianza cosmica (ineliminabile e importante a grandi scale angolari) che gli errori statistici e di calibrazione. La linea continua rappresenta il best fit con un modello CDM a 6 parametri liberi, la banda verde rappresenta la varianza cosmica aspettata. Sono visibili ben 7 picchi dovuti alle oscillazioni acustiche del plasma primordiale. 
Il colpo d'occhio della Fig. 8 indica quello che si può considerare il risultato più straordinario di Planck, e cioè il livello incredibile di precisione con cui i dati descrivono il semplice modello a sei parametri. Per la prima volta le misure di un singolo esperimento coprono tutti i multipoli significativi $(2<\ell<2500)$ con precisione limitata dalla varianza cosmica, dal residuo ineliminabile dei foreground e dal Silk damping. Questo realizza l'obiettivo di una misura definitiva delle anisotropie in temperatura e consente una stima di alta precisione dei parametri cosmologici, i cui valori sono riportati in Tab. 2. Tali valori risultano stabili rispetto a diverse procedure di separazione delle componenti e non sono significativamente affetti da errori sistematici.

Nell'analisi che ha portato ai risultati in Tab. 2 si è anche tenuto conto dell'effetto del "lensing gravitazionale" causato dalla massa delle strutture cosmiche (principalmente materia oscura) incontrate dai fotoni CMB nel loro lungo viaggio dalla superficie di ultimo scattering fino al telescopio di Planck. Il fenomeno del lensing riguarda le lievi deviazioni dalla traiettoria lineare che i fotoni subiscono a causa della deformazione locale dello spazio associata alla presenza di massa (come previsto dalla relatività generale). L'effetto sullo spettro di potenza è quello di smussare leggermente la struttura dei picchi acustici. I dati di Planck sono così precisi che questo fenomeno è rivelato con un livello altissimo di confidenza. Da questo studio è possibile ricavare l'integrale del potenziale gravitazionale lungo la linea di vista, e quindi la distribuzione di materia oscura nell'universo. L'ampiezza dello spettro di potenza del potenziale gravitazionale così valutato è perfettamente consistente con quella aspettata dall'evoluzione delle perturbazioni iniziali di densità responsabili delle anisotropie primarie della CMB. Il loro rapporto è misurato in $0.99 \pm 0.05$, una evidenza a $20 \sigma$ dell'esistenza di lensing gravitazionale sui fotoni CMB. Le informazioni che Planck ha ottenuto sul lensing contribuiscono a risolvere degenerazioni tra parametri, e combinate con quelle dello spettro di potenza contribuiscono alla precisione con cui si ottengono i valori dei parametri cosmologici.

Le misure riportate in Tab. 2 segnano un nuovo livello di precisione in cosmologia. L'orizzonte acustico alla ricombinazione, dedotto dalla scala angolare delle anisotropie predominanti nella mappa CMB, è determinato con precisione dello $0.1 \%$. Lo spettro iniziale delle perturbazioni di densità è consistente su tutto lo spettro (oltre tre decadi) con un singolo indice spettrale $n_{s}$, il cui valore è vicino all'unità (invarianza di scala) ma leggermente minore. Lo scostamento dall'unità del 
$23.5 \%$ è determinato con un livello di confidenza di $6 \sigma$. Tutto ciò è in linea con lo scenario previsto dai modelli di inflazione. Le misure consentono di escludere un certo numero di modelli inflazionari tra i molti ipotizzati dai teorici. L'ampiezza $A_{s}$ è determinata dalla calibrazione globale dello spettro. I dati di Planck confermano poi che la densità totale di materia-energia nell'universo è dominata da materia oscura e energia oscura, tanto che insieme esse costituiscono quasi il 95\% della densità totale. I parametri di densità misurati da Planck sono più precisi e leggermente diversi da quelli determinati da misure precedenti. In particolare rispetto a WMAP i risultati di Planck (considerando i soli dati di $\mathrm{CMB})$ mostrano che il contributo dell'energia oscura è più basso del $6 \%$, quello dei barioni è più alto del $9 \%$, e quello della materia oscura è più alto del 18\%. Queste variazioni si riducono di un fattore 3 quando si includono nei due fit anche dati di oscillazioni acustiche barioniche. Lo spessore ottico $\tau$, qui determinato dai soli dati di anisotropia di temperatura, potrà essere misurato con miglior precisione grazie all'analisi della polarizzazione.

Tab. 2 - I valori dei 6 parametri cosmologici indipendenti misurati da Planck, ottenuti tramite una procedura di best fit dello spettro di potenza di Fig. 9 e del lensing gravitazionale, usando il modello CDM (Planck Collaboration 2014).

\begin{tabular}{ccc} 
& \multicolumn{2}{c}{ Planck (CMB+lensing) } \\
\cline { 2 - 3 } Parameter & Best fit & $68 \%$ limits \\
\hline$\Omega_{\mathrm{b}} h^{2} \ldots \ldots \ldots \ldots$ & 0.022242 & $0.02217 \pm 0.00033$ \\
$\Omega_{\mathrm{c}} h^{2} \ldots \ldots \ldots \ldots$ & 0.11805 & $0.1186 \pm 0.0031$ \\
$100 \theta_{\mathrm{MC}} \ldots \ldots \ldots$ & 1.04150 & $1.04141 \pm 0.00067$ \\
$\tau \ldots \ldots \ldots$ & 0.0949 & $0.089 \pm 0.032$ \\
$n_{\mathrm{s}} \ldots \ldots \ldots$ & 0.9675 & $0.9635 \pm 0.0094$ \\
$\ln \left(10^{10} A_{\mathrm{s}}\right) \ldots \ldots \ldots$ & 3.098 & $3.085 \pm 0.057$ \\
\hline
\end{tabular}

Dai parametri di base della Tab. 2 se ne deducono altri, come ad esempio la costante di Hubble. La misura di Planck, $H_{0}=67.9 \pm 1.5 \mathrm{~km}$ $\mathrm{s}^{-1} \mathrm{Mpc}^{-1}$, è consistente con quella trovata da WMAP e due volte più precisa. Il suo valore è relativamente basso, in ottimo accordo con recenti misure statistiche di distribuzione su larga scala (oscillazioni acustiche barioniche, BAO). Tuttavia esso si discosta per $2.5 \sigma$ dal valore ottenuto con i metodi classici di correlazione fra distanze e red- 
shift nell'universo (relativamente) vicino, una tensione che sarà importante investigare utilizzando i dati dell'intera missione. Combinando il valore della costante di Hubble e dei parametri di densità, e utilizzando la soluzione alle equazioni di Friedmann della relatività generale, otteniamo la stima dell'età cosmica pari a $t_{0}=13.80 \pm 0.06$ miliardi di anni: è una misura con precisione dello $0.4 \%$ della "data di nascita" dell'universo.

\section{OLTRE IL MODELLO STANDARD?}

Ma possiamo trovare qualche significativo miglioramento nel fit estendendo il modello con l'aggiunta di ulteriori parametri liberi? La risposta al momento è negativa. La più ovvia generalizzazione del modello standard è rilassare l'assunzione di curvatura nulla, $\Omega_{K}=0$. Eliminando questo vincolo e utilizzando le misure di Planck si possono porre limiti superiori assai stringenti al parametro di curvatura. Usando i dati di Planck (sia CMB che lensing), insieme a SPT e ACT per minimizzare gli effetti di foreground su piccola scala angolare, si ottiene un limite $\Omega_{K}=-0.0096_{-0.0082}^{+0.010}$.

Uno degli aspetti più interessanti che le misure di Planck possono sondare oltre il modello standard è l'eventuale esistenza di una quarta specie di neutrino e il limite alla massa di queste particelle. L'analisi mostra che non c'è evidenza di altre specie di particelle relativistiche (oltre ai fotoni e alle 3 specie di neutrini). Combinando i dati di Planck con altri dati $\mathrm{CMB}$ e $\mathrm{BAO}$ si ottiene un limite assai stringente per la somma delle masse dei neutrini, pari a $\sum m_{v}<0.23 \mathrm{eV}$.

Altri importanti fronti di analisi oltre il modello standard riguardano l'isotropia globale e la gaussianità delle fluttuazioni. Come già sottolineato, i modelli di inflazione prevedono che le fluttuazioni di densità iniziali che producono le anisotropie siano originate da fluttuazioni quantistiche, espanse dal processo di inflazione. In questo caso ci si aspetta che le fluttuazioni siano isotrope, e con (eventuali) deviazioni bassissime dalla distribuzione Gaussiana, almeno per i modelli di inflazione più semplici. La mappa della $\mathrm{CMB}$ misurata da Planck è stata analizzata a fondo per investigare queste due caratteristiche.

Per quanto riguarda l'isotropia, i dati di Planck mostrano cenni di deviazioni che persistono indipendentemente dal metodo di separazione delle componenti, dalla maschera di esclusione dell'emissione galat- 
tica, dalla combinazione di mappe utilizzata. Queste anomalie erano state evidenziate anche da WMAP, e ora i nuovi dati di Planck dimostrano che esse non derivano da effetti sistematici o da residui di foreground. Le più importanti sono un allineamento tra le componenti di quadrupolo e di ottupolo dell'anisotropia, un'asimmetria di potenza tra emisfero nord ed emisfero sud galattici, e la presenza di un decremento significativo della brillanza, detta "cold spot". Il livello di significatività di queste deviazioni è marginale, circa $3 \sigma$, e al momento non è chiaro se si tratti di fluttuazioni statistiche o del segno di qualcosa più profondo. Non si trova invece alcuna evidenza di non-gaussianità delle fluttuazioni. Il parametro che descrive la lieve non-gaussianità prodotta nei modelli inflazionari, $f_{N L}$, misura l'ampiezza della non linearità di tipo quadratico nel modo di perturbazione di curvatura. La mappa di Planck è stata analizzata con diversi estimatori, e vincola il parametro $f_{N L}$ ad essere nullo con limiti superiori molto più stringenti di quelli ottenuti da tutte le misure precedenti.

\section{CONCLUSIONI}

Dopo oltre due decenni di design, sviluppo, testing, osservazioni e analisi dati, i primi risultati cosmologici di Planck ricompensano gli sforzi prodotti nell'arco di questo entusiasmante progetto. La missione Planck è il risultato di una vasta collaborazione internazionale (Fig. 10) che ha visto il contributo di centinaia di scienziati e ingegneri di Europa, USA e Canada, e nella quale l'Italia ha giocato un ruolo di primissimo piano. Le osservazioni di precisione di Planck forniscono 9 mappe di tutto il cielo a frequenze tra 30 e $857 \mathrm{GHz}$. Le mappe che sono state qui discusse riguardano i primi 15.5 mesi di osservazioni, mentre i risultati della missione completa (inclusa l'analisi di polarizzazione) è attualmente in corso.

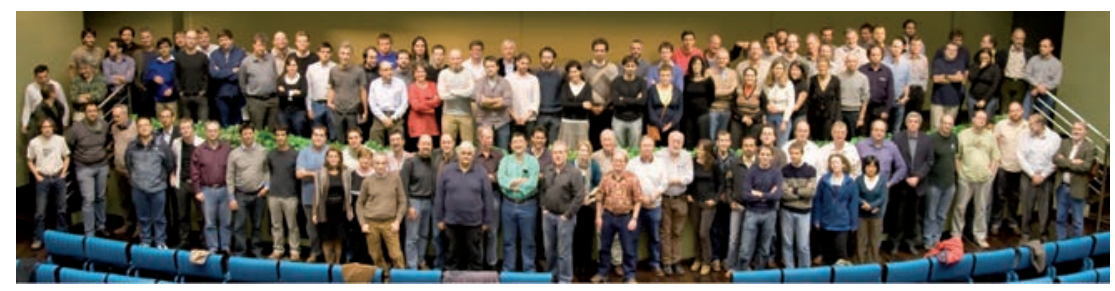

Fig. 10 - Foto di gruppo del Planck Joint Core Team. 
Lo spettro di potenza misurato è perfettamente consistente con il modello standard CDM, nel quale la densità di massa-energia dell'universo è dominata da energia e materia oscura, con una componente sub-dominante di materia barionica. Inoltre, in linea con quanto previsto dal processo inflazionario, le fluttuazioni di densità iniziali sono gaussiane e approssimativamente invarianti di scala. I dati non lasciano molto margine a evidenze di nuova fisica, come una quarta specie di neutrino o modelli anisotropi. I parametri cosmologici sono misurati con grande accuratezza $(\sim 1 \%)$, spingendo la cosmologia di precisione in una nuova fase.

Il modello standard ne esce fortemente rafforzato. D'altra parte non dobbiamo dimenticare che, paradossalmente, il $95 \%$ di ciò che rientra nel modello cosmologico standard CDM è "dark", nel senso che ancora non ne comprendiamo la natura fisica. Energia oscura $(\Lambda)$ e materia oscura $\mathrm{CDM}$ ) infatti rimangono al momento estranee all'altro grande "modello standard" della fisica, quello delle particelle elementari, che dà ragione solamente di un esiguo $4.9 \%$ di materia barionica.

Ma è un dato di fatto che con sei soli parametri riusciamo a dar conto della fenomenologia dell'universo primordiale basata sulla fisica lineare delle oscillazioni acustiche attivate da fluttuazioni primordiali. L'universo iniziale era tanto semplice da essere ben descritto da sei numeri. Questo ci impressiona e ci affascina se pensiamo che da quella realtà semplicissima, elementare e quasi indistinta si è originata la varietà di strutture che osserviamo nell'universo attuale, capace di raggiungere localmente livelli impensabili di complessità come quelli che caratterizzano gli organismi biologici, fino alla vita cosciente.

\section{BIBLIOGRAFIA}

Bersanelli, M. et al., 1996, Report on the Phase A Study of COBRAS/SAMBA, ESA Publication D/SCI(96)3.

Bersanelli, M. et al., 2010, Planck pre-launch status: Design and description of the Low Frequency Instrument, Astronomy and Astrophysics, 520, A4.

Boyle, et al., 2006 Inflationary Predictions for Scalar and Tensor Fluctuations Reconsidered, Physical Review Letters, vol. 96, Issue 11, id. 111301.

Das, S. et al., 2014, The Atacama Cosmology Telescope: Temperature and Gravitational Lensing Power Spectrum Measurements from Three Seasons of Data, 2014, JCAP, 04, 014D.

De Bernardis, P. et al., 2000, A flat universe from bigh-resolution maps of the cosmic microwave background radiation, Nature, 404, 955. 
Guth, A.H., The inflationary universe. The quest for a new theory of cosmic origins, Addison-Wesley, 1997.

Hinshaw, G.F. et al., 2013, Nine-Year Wilkinson Microwave Anisotropy Probe (WMAP) Observations: Cosmology Results ApJS., 208, 19H

Hubble, E., 1929, A relation between distance and radial velocity among extra-galactic nebulae, Proc. National Acad. Sci. 15, Issue 3, p. 168.

Kovac, J. et al., 2002, Detection of polarization in the cosmic microwave background using DASI, Nature, 420, 772.

Lamarre, J.-M. et al., 2010, Planck pre-launch status: The HFI instrument, from specification to actual performance, Astronomy and Astrophysics, 520, A9.

Lange, A.E. et al., 2001, First Estimations of Cosmological Parameters From Boomerang, Phys. Rev. D, 63, 042001.

Lemaître, G., 1927, Un univers bomogène de masse constante et de rayon croissant, rendant compte de la vitesse radiale des nébuleuses extra-galactiques, Ann. Soc. Sci. de Bruxelles, 47, 49.

Mather, J. et al., 1999, Calibrator Design for the COBE Far-Infrared Absolute Spectrophotometer (FIRAS), The Astrophysical Journal, vol. 512, pp. 511-520.

Penzias, A. and Wilson, R., 1965, A Measurement of Excess Antenna Temperature at $4080 \mathrm{Mc} / \mathrm{s}$. Astrophysical Journal, vol. 142, p. 419-421.

Percival, W.J. et al., 2002, Parameter constraints for flat cosmologies from cosmic microwave background and 2dFGRS power spectra, Monthly Noices of the Royal Astronomical Society, 337, 1068-1080.

Planck Collaboration, 2005, The Planck Collaboration 2005, "Planck: The Scientific Programme”, ESA publication ESA-SCI(2005)/01.

Planck Collaboration, 2011a, Planck early results. I. The Planck mission, Astronomy and Astrophysics, 536, A1, 2011.

Planck Collaboration, 2011b, Planck early results. II. The thermal performance of Planck, Astronomy and Astrophysics, 536, A2.

Reichardt, C.L., et al., 2012, A measurement of secondary cosmic microwave background anisotropies with two years of South Pole Telescope observations, ApJ, 755, 70.

Smoot, G.F. et al., 1994, Astrophysical Journal, 396 (1992) L1; Mather J.C. et al., Astrophysical Journal, 420, 439.

York, D.G. et al., 2000, The Sloan Digital Sky Survey: Technical Summary; The Astronomical Journal, 120, 1579. 